\title{
Importance of Intermolecular Interactions in Assessing Hopping Mobilities in Organic Field Effect Transistors: Pentacene versus Dithiophene-tetrathiafulvalene
}

\author{
Stefan T. Bromley, ${ }^{*}$, Marta Mas-Torrent, ${ }^{*}, \neq$ Peter Hadley, ${ }^{\ddagger}$ and Concepció Rovira ${ }^{\S}$ \\ Ceramic Membrane Centre, The Pore, DelftChemTech, Delft University of Technology, Julianalaan 136, \\ 2628 BL Delft, The Netherlands, Kavli Institute of NanoScience, Delft University of Technology, \\ Lorentzweg 1, 2628 CJ Delft, The Netherlands, and Institut de Ciència de Materials de Barcelona, \\ Campus de la Universitat Autònoma de Barcelona, 08193 Bellaterra, Spain
}

Received January 14, 2004; E-mail: s.t.bromley@tnw.tudelft.nl; marta@qt.tn.tudelft.nl

A growing interest in organic field effect transistors (OFETs) has emerged in past years due to their potential applications in electronics where low-cost, large area coverage, and structural flexibility are required. ${ }^{1}$ OFET single crystals are found to give the highest mobilities largely due to their regular molecular ordering that permits extensive intermolecular orbital overlap to occur. Crystalline pentacene is probably the most widely studied organic semiconductor and, because of its high performance (hole mobility of $\left.1.5 \mathrm{~cm}^{2} /(\mathrm{V} \cdot \mathrm{s})\right),{ }^{2}$ has been the benchmark by which other OFETs are measured. In this paper, we report on a theoretical study to understand the high mobility found in dithiophene-tetrathiafulvalene (DT-TTF) transistors, ${ }^{3}$ with respect to that known for pentacene, using an extended measure of the reorganization energy. We demonstrate that the molecular packing is a key factor in assessing hopping mobilities. The relationship between crystal structure and transport properties of a material, crucial to understand for the rational design of new OFET materials, is thus elucidated.

At room temperature, the charge mobility of organic materials is often determined by a hopping transport process, which can be depicted as an electron or hole transfer reaction in which an electron or hole is transferred from one molecule to the neighboring one. The localization of charge on a molecule for a sufficient time allows the nuclei to adopt the optimal geometry of the charged state, ${ }^{4}$ coupling molecular relaxation with the charge mobility. Two major parameters determine self-exchange rates and, thus, the charge mobility: ${ }^{5}$ (i) the electronic coupling between adjacent molecules (transfer integral), ${ }^{6}$ which needs to be maximized, and (ii) the reorganization energy $\left(\lambda_{\text {reorg }}\right)$, which needs to be small for efficient charge transport. Neglecting the contributions due to the medium polarization and molecular vibrations, in a hole-hopping material, $\lambda_{\text {reorg }}$ corresponds to the sum of two relaxation energies, $\lambda_{\text {rel }}{ }^{(1,2)}$, for the transformation of one molecule from the neutral state to the +1 charged state, and, for a neighboring molecule, the transformation from the charged state to the neutral molecular state. These two portions are typically nearly identical $\left(\lambda_{\text {reorg }} \approx 2 \lambda_{\text {rel }}\right){ }^{7}$ The reorganization energy gives a measure of the energy loss (or hopping efficiency) of a charge carrier passing through a single molecule. To explain the high mobility of pentacene transistors, previous studies have focused on the reorganization energy of the isolated pentacene molecule. ${ }^{5}$ Interestingly, the reorganization energy calculated for the pentacene molecule is extremely low $(0.098 \mathrm{eV}),{ }^{5}$ providing persuasive evidence for its high hole mobility.

Ceramic Membrane Centre, DelftChemTech, TUDelft.

Kavli Institute of Nanoscience, TUDelft.

$\S$ Institut de Ciència de Materials de Barcelona.
Chart 1
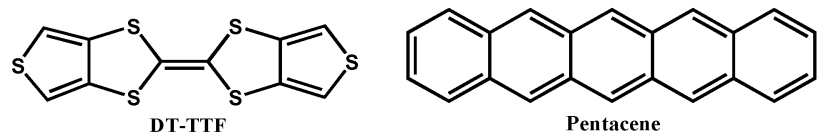

Table 1. $\lambda_{\text {reorg }}$ Values (eV) for Pentacene and DT-TTF

\begin{tabular}{lll}
\hline & pentacene & DT-TTF \\
\hline isolated (boat geom.) & & 0.574 \\
isolated (planar geom.) & 0.098 & 0.238 \\
embedded molecule & $0.080(0.32|\mathrm{e}|)^{a}$ & $0.042(0.28|\mathrm{e}|)^{a}$ \\
\hline
\end{tabular}

${ }^{a}$ Total charge on the charged embedded molecule.

Most attention for improving the mobility of OFETs has been placed on the development of improved device fabrication techniques. ${ }^{8}$ Other feasible strategies have attempted to increase the relatively small intermolecular orbital overlap found in pentacene by directed functionalization, ${ }^{9}$ or by searching for new materials with high intermolecular electronic coupling and low $\lambda$. Recently, we reported a very high mobility of $1.4 \mathrm{~cm}^{2} /(\mathrm{V} \cdot \mathrm{s})$ for a DT-TTF single-crystal transistor prepared using a simple room-temperature drop casting technique. ${ }^{3}$ Similar to pentacene, DT-TTF is a small conjugated, symmetric molecule (Chart 1). The crystal packing of DT-TTF is also similar to that of pentacene, ${ }^{10}$ although in DTTTF there is a shifted-cofacial $\pi$-stacking as compared to a tilted $\pi$-stacking in pentacene. The outstanding device performance and the fact that DT-TTF can be easily processed opens new perspectives in the field of OFETs.

Considerable attention has been placed in finding correlations between $\lambda$ and hole mobility for different compounds such as triphenylamine derivatives, biphenyl, and various compounds formed by fused aromatic rings. ${ }^{11-13}$ The extremely low reorganization energy of pentacene, even compared to substituted pentacenes, was attributed to its higher symmetry and resultant more delocalized nature of the orbital from which an electron is removed. ${ }^{5}$ We have performed density functional calculations to calculate $\lambda_{\text {reorg }}$ for the isolated DT-TTF molecule. All reported calculations were performed at a $6-31 \mathrm{G}(\mathrm{d}, \mathrm{p}) / \mathrm{B} 3 \mathrm{LYP} \mathrm{P}^{14}$ level of theory using the GAMESS-UK ${ }^{15}$ code. As reported before for similar TTF derivatives, ${ }^{16}$ the DT-TTF molecule adopts a distorted boat conformation in the neutral ground state, but a planar conformation for the +1 charged state. However, as it is also known that neutral DT-TTF molecules crystallize with a planar conformation, ${ }^{17}$ having a slightly higher energy than the boat conformation, we have calculated $\lambda_{\text {reorg }}$ employing both planar and boat neutral conformations (Table 1). The resultant $\lambda_{\text {reorg }}$ while keeping the molecule planar in the neutral state is significantly lower than the respective $\lambda_{\text {reorg }}$ value using 


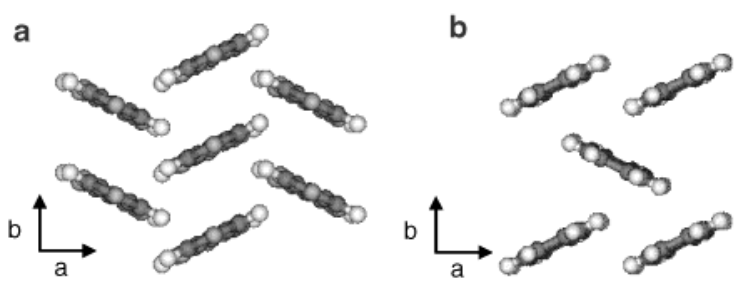

Figure 1. Molecular crystal ordering in (a) DT-TTF and (b) pentacene.

the neutral boat conformation, consistent with the fact that the +1 charged molecule is also planar. The $\lambda_{\text {reorg }}$ value for the isolated planar DT-TTF molecule is also similar to that of other studied molecules, ${ }^{7}$ but still over double of that calculated for pentacene. Considering both compounds have transfer integrals of the same order (see Supporting Information), their reported similarly high mobilities cannot be rationalized by the isolated molecular $\lambda_{\text {reorg }}$ values.

The lowering of $\lambda_{\text {reorg }}$ for the more constrained planar DT-TTF molecule indicates that the local environmental steric packing of nearest-neighboring $(\mathrm{NN})$ molecules may play an important role in maximizing the mobility of the DT-TTF crystal. Usually, reorganization energies are calculated with respect to isolated ground-state molecules, with the calculated results often compared to gas-phase photoelectron spectra. ${ }^{5 b}$ Such studies explicitly exclude the electronic coupling between adjacent molecules in the crystal and, thus, may not always provide a good measure of relative crystal mobilities. Herein, we further calculated $\lambda_{\text {reorg }}$ of molecules while also explicitly considering how they interact with, and are constrained by, their specific local crystal packing. We consider DT-TTF and pentacene molecules both embedded by NN molecules taken in the primary charge conduction directions of the respective crystals. For DT-TTF, this entails a stack of three molecules along the $b$ axis (Figure 1a), and for pentacene, ${ }^{18}$ this entails an array of five molecules encompassing both $a+b$ and $a-b$ crystal axes (Figure 1b). ${ }^{10 a}$ In each case, the peripheral molecules were fixed at the geometry of the respective crystal, while the embedded molecule was allowed to relax. In the case of the embedded pentacene molecule, we find there is very little difference in $\lambda_{\text {reorg }}$ with respect to an isolated pentacene molecule, indicating a weak dependence on intermolecular interactions. For the embedded DT-TTF molecule, however, a $\lambda_{\text {reorg }}$ of only $0.042 \mathrm{eV}$ was obtained. This latter result is particularly surprising when comparing it to the $\lambda_{\text {reorg }}$ for an isolated planar DT-TTF molecule $\left(\lambda_{\text {reorg }}\right.$ is found to be $82 \%$ lower in the embedded molecule), showing that, in addition to the steric constraint of the crystal packing keeping the neutral molecule planar, $\lambda_{\text {reorg }}$ has a strong dependence on the local intermolecular coupling. Comparing the embedded results, DT-TTF has $\lambda_{\text {reorg }}$ values of the same order of that of pentacene, which would help to explain the high mobility reported for DT-TTF single-crystal transistors. ${ }^{3}$

In both pentacene and planar DT-TTF, the molecular geometries of the isolated molecules in the neutral state are almost identical to those of the respective embedded molecules. Comparing the corresponding embedded and isolated charged molecules, however, shows that although for pentacene there are small differences in bond lengths (max. $0.009 \AA$ ), for DT-TTF the embedded molecule has a significantly shorter central $\mathrm{C}=\mathrm{C}$ bond $(-0.040 \AA)$ and shorter adjoining $\mathrm{C}-\mathrm{S}$ bonds $(-0.028 \AA)$. This intermolecular-induced structural change further reduces the geometric relaxation needed going between charged and neutral molecules and thus also $\lambda_{\text {reorg. }}$. Calculations for an isolated DT-TTF molecule using the embedded geometries $\left(\lambda_{\text {reorg }}=0.094 \mathrm{eV}\right)$ confirm that the $\lambda_{\text {reorg }}$ decrease is predominantly due to this structural change, which is likely caused by local intermolecular charge transfer from $\mathrm{NN}$ molecules to the central DT-TTF molecule assisted by the S $\cdots S$ intermolecular interactions. This is supported by summing atomic charges on the embedded charged molecule which reveals a $\sim 72 \%$ decrease in the total charge. For the more rigid pentacene molecule, a similar charge-transfer effect is observed but without the accompanying

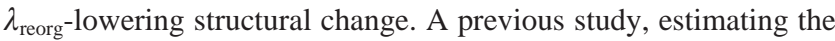
contribution of orbital polarization and charge transfer of $\mathrm{NN}$ molecules to the total reorganization energy of molecular polarons in anthracene, employing a similar approach (fixed NN pairs of molecules) as used herein, also resulted in a comparably small reduction of $\lambda_{\text {reorg }}$ as we observe for pentacene. ${ }^{19}$ Contrary to most other experimental and theoretical approaches, our extended methodology yields $\lambda_{\text {reorg }}$ for a molecule together with the stabilizing effect of local intermolecular interactions.

In conclusion, our calculations of the reorganization energies of both pentacene and DT-TTF molecules, including intermolecular interactions, yield very low $\lambda_{\text {reorg }}$ values, helping to explain the high mobilities reported for these crystals. We clearly demonstrate that local intermolecular interactions can have a strong influence on $\lambda_{\text {reorg }}$ values and, thus, hopping mobilities. This result is of high importance for the design of new OFET materials.

Acknowledgment. M.M.-T. and C.R. thank Generalitat de Catalunya and DGI:Spain/BQU2003-00760 for financial support.

Supporting Information Available: Computational methods and detailed results. This material is available free of charge via the Internet at http://pubs.acs.org.

\section{References}

(1) Dimitrakopoulos, C. D.; Malenfant, P. R. L. Adv. Mater. 2002, 14, 99117.

(2) Nelson, S. F.; Lin, Y.-Y.; Gundlach, D. J.; Jackson, T. N. Appl. Phys. Lett. 1998, 72, 1854-1856.

(3) Mas-Torrent, M.; Durkut, M.; Hadley, P.; Ribas, X.; Rovira C. J. Am. Chem. Soc. 2004, 126, 984-985.

(4) Duke, C. B.; Schein, L. Phys. Today 1980, February, 42.

(5) (a) Marcus, R. A. Rev. Mod. Phys. 1993, 65, 599. (b) Gruhn, N. E.; da Silva Filho, D. A.; Bill, T. G.; Malagoli, M.; Coropceanu, V.; Kahn, A.; Brédas, J.-L. J. Am. Chem. Soc. 2002, 124, 7918-7919.

(6) Brédas, J. L.; Calbert, J. P.; da Silvo Filho, D. A.; Cornil, J. Proc. Natl. Acad. Sci. U.S.A. 2002, 99, 5804-5809.

(7) Malagoli, M.; Brédas, J. L. Chem. Phys. Lett. 2000, 327, 13-17.

(8) Lin, Y.; Gundlach, D. J.; Nelson, S. F.; Jackson, T. N. IEEE Trans. Electron Devices 1997, 44, 1325-1331.

(9) (a) Anthony, J. E.; Brooks, J. S.; Eaton, D. L.; Parkin, S. R. J. Am. Chem. Soc. 2001, 123, 9482-9483. (b) Anthony, J. E.; Eaton, D. L.; Parkin, S. R. Org. Lett. 2002, 4, 15-18.

(10) (a) Cornil, J.; Calbert, J. Ph.; Brédas, J. L. J. Am. Chem. Soc. 2001, 123, 1250-1251. (b) Holmes, D.; Kumaraswamy, S.; Matzger, A. J.; Vollhardt, K. P. C. Chem.-Eur. J. 1999, 5, 3399-3412.

(11) Amashukeli, X.; Winkler, J. R.; Gray, H. B.; Gruhn, N. E.; Lichtenberger, D. L. J. Phys. Chem. A 2002, 106, 7593-7598.

(12) Sakanoue, K.; Motoda, M.; Sugimoto, M.; Sakaki, S. J. Phys. Chem. A 1999, 103, 5551-5556.

(13) Lin, B. C.; Cheng, C. P.; Lao, Z. P. M. J. Phys. Chem. A 2003, 107, $5241-5251$

(14) Becke, A. D. J. Phys. Chem. 1993, 98, 5648

(15) GAMESS-UK is a package of ab initio programs by Guest, M. F.; van Lenthe, J. H.; Kendrick, J.; Sherwood, P., with contributions from Amos, R. D.; Buenker, R. J.; van Dam, H.; Dupuis, M.; Handy, N. C.; Hillier, I. H.; Knowles, P. J.; Bonacic-Koutecky, V.; von Niessen, W.; Harrison, R. J.; Rendell, A. P.; Saunders, V. R.; Schoffel, K.; Stone, A. J.; Tozer, D.

(16) Demiralp, E.; Goddard, W. A., III. J. Phys. Chem. A 1997, 101, 81288131.

(17) Rovira, C.; Veciana, J.; Santaló, N.; Tarrés, J.; Cirujeda, J.; Molins, E.; Llorca, J.; Espinosa, E. J. Org. Chem. 1994, 59, 3307-3313.

(18) Crystal structure from Cambridge Database (ref no.: CCDC-114447).

(19) Silinsh, E. A.; Klimkāns, A.; Larsson, S.; Č́pek, V. Chem. Phys. 1995, $198,311-331$

JA049762A 